ISSN e- 2594-1100

en-claves del pensamiento / núm. 31 / enero-junio / 2022 / e504

https://doi.org/10.46530/ecdp.v0i31.504 DOSSIER

enn-claves

\title{
INCLUSIÓN DEL ALUMNADO CON TEA EN LOS TIEMPOS DE RECREO
}

\author{
Inclusion of Students with ASD in Recess
}

\author{
Carlos García Junco, Universidad Internacional de la Rioja, España \\ Correo electrónico: carlos.garciajunco@unir.net \\ Recibido: $15 / 05 / 2021$ \\ Aceptado: $15 / 11 / 2021$ \\ Publicado: 07/01/2022
}

\begin{abstract}
Resumen. Las dificultades comunicativas y en habilidades sociales que presenta gran parte del alumnado con TEA (Trastorno del Espectro Autista), sumado a sus dificultades académicas, hacen que sean niños con altos porcentajes de fracaso escolar, además de sumarse otros factores de riesgo social: la exclusión social y el acoso escolar o bullying. Mediante la introducción de diversos juegos y apoyos en el recreo, se busca revertir la situación de aislamiento que sufren diariamente niños y niñas durante su jornada educativa, aumentando de manera gradual los tiempos de interacción positiva de los mismos. Utilizando la metodología observacional se crea un sistema de registro de conductas observadas durante los recreos, con el objetivo de registrar y evaluar las conductas del alumnado. Los resultados obtenidos han sido muy satisfactorios, demostrando que la introducción de juegos en el recreo, aumenta los tiempos de juego y de interacción positiva en el alumnado con TEA, así como en el resto de alumnado que presenta dificultades de relación y/o conductas disruptivas.

Palabras clave: inclusión, TEA, juegos, recreo, vulnerabilidad.
\end{abstract}

Abstract. The communication and social skills difficulties that a large part of the students with ASD (Autism Spectrum Disorder) present, added to their academic difficulties, make them children with high rates of school failure, in addition to other social risk factors: exclusion social and bullying. By introducing various games and supports at recess, the aim is to reverse the isolation situation that boys and girls suffer daily during their educational journey, gradually increasing their positive interaction times. Using the observational methodology, a system for recording behaviors observed during breaks is created, with the aim of recording and evaluating the behaviors of students. The results obtained have been very satisfactory, showing that the introduction of games at recess increases play times and positive interaction times in students with ASD, as well as in the rest of the students who present relationship difficulties and / or disruptive behaviors.

Keywords: inclusion, ASD, play times, recess times, vulnerability.

Cómo citar: García Junco, C. (2022). Inclusión del alumnado con TEA en los tiempos de recreo. ENCLAVES del pensamiento, 0(31), e504. doi: https://doi.org/10.46530/ecdp.v0i31.504

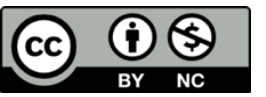

Esta obra está protegida bajo una Licencia

Creative Commons Atribución - No comercial

4.0 Internacional 


\section{Introducción}

Todas las personas somos diferentes ${ }^{1}$ y por ley, la educación inclusiva en España, ${ }^{2}$ es un derecho de todos los alumnos y alumnas. ${ }^{3}$ La tendencia educativa actual consiste en caminar hacia una educación inclusiva, en la que todos los niños aprenden, ${ }^{4}$ pero se siguen observando múltiples casos de exclusión social y acoso escolar. ${ }^{5}$ Se plantea, por tanto, la necesidad de intervenir, ya que continúan existiendo actitudes negativas hacia las personas con discapacidad ${ }^{6}$ que dificultan o que impiden alcanzar una inclusión total en el ámbito educativo, ${ }^{7}$ además de observarse que sigue existiendo una tendencia a escolarizar a los niños y niñas con diversidad funcional en centros educativos específicos. ${ }^{8}$

En el ámbito educativo, el patio de recreo es un espacio de descanso y diversión, ${ }^{9}$ pero también de riesgo de exclusión social. ${ }^{10}$ El recreo es un espacio educativo en el que prima el juego libre, ${ }^{11}$ pero algunos niños y niñas, por diversas circunstancias, no juegan o no interaccionan de manera adecuada con sus compañeros, ${ }^{12}$ apareciendo conductas disruptivas y de aislamiento en el patio que pueden dar lugar a situaciones de acoso escolar o bullying. ${ }^{13}$

${ }^{1}$ Belkys Edith Torres, Sistema CEDA: Evaluación alternativa para una enseñanza inclusiva" (Oviedo: Universidad de Oviedo, 2017).

${ }^{2}$ Gerardo Echeita e Ignacio Calderón-Almendros, "Obstáculos a la inclusión: cuestionando concepciones y prácticas de evaluación psicopedagógica”, Ámbitos de Psicopedagogía y Orientación, núm. 41 (noviembre 2014): 1-11.

${ }^{3}$ Gerardo Echeita y Cecilia Simón, "Comprender la educación inclusiva para intentar llevarla a la práctica", en Henar Rodríguez y Luis Torrego (coords.), Educación inclusiva, equidad y derecho a la diferencia (Madrid: Wolters Kluwer, 2013), 33-65.

${ }^{4}$ César Bona, Las escuelas que cambian el mundo (Barcelona: Plaza \& Janés, 2016).

${ }^{5}$ Pedro María Uruñuela, Trabajar la convivencia en los centros educativos (Madrid: Narcea, 2016).

${ }^{6}$ María Ángeles Alcedo et al., "Eficacia del contacto e información como técnicas de cambio de actitudes hacia personas con discapacidad en niños de Educación Primaria", Universitas Psychologica, núm. 12 (agosto, 2012): 493-504. https://doi.org/10.11144/Javeriana.UPSY12-2.ecit.

${ }^{7}$ Francisco González-Gil et al., "Percepciones del profesorado sobre la inclusión: estudio preliminar", Revista Electrónica de Formación del Profesorado, núm. 19 (abril, 2016): 11-24. doi: https://doi.org/10.6018/reifop.19.3.219321.

${ }^{8}$ Ignacio Calderón y Paula Verde, Reconocer la diversidad (Barcelona: Octaedro, 2018).

9 Pablo Gras y Jorge Paredes, "El recreo ¿Sólo para jugar?”, Revista Digital de Educación Física, núm. 36, (octubre, 2015): 18-27.

10 Jenny María Artavia, "Manifestaciones ocultas de violencia, durante el desarrollo del recreo escolar", Revista Electrónica Actualidades Investigativas en Educación, núm. 13 (enero, 2013): 1-21.

${ }^{11}$ Gey Lagar, Patios y parques dinámicos (Oviedo: Trabe, 2015).

12 Carlos García, ¿A qué jugamos? Inclusión del alumnado con TEA en el tiempo de recreo en centros escolares (Valencia: Psylicom, 2018).

${ }^{13}$ Lydia Nacimiento y Joaquín Antonio Mora, Estrategias de afrontamiento ante bullying y cyberbullying: condicionantes y consecuencias (Sevilla: Universidad de Sevilla, 2018). 
Por otro lado, está la importancia del juego en el desarrollo de los niños y niñas. ${ }^{14}$ El juego es una actividad humana compleja, ${ }^{15}$ que ha estado presente a lo largo de toda la historia de la humanidad. ${ }^{16}$ Con el juego se despliegan aspectos muy importantes relacionados con el desarrollo social, cognitivo y emocional. ${ }^{17}$ El juego es muy importante para el crecimiento del ser humano, ${ }^{18}$ lo cual nos hace pensar que cualquier niño o niña que no pueda acceder al juego compartido, tanto en el ámbito educativo, como social, podrá tener menos oportunidades de desarrollo y estimulación; pudiendo ser un factor desencadenante de exclusión. El juego es un medio canalizador de sentimientos y expresiones, ${ }^{19}$ que a su vez facilita el progreso en múltiples áreas como puede ser el componente social, del lenguaje, conductual, etc. El juego aparece en los niños y las niñas desde edades tempranas, es más, hay investigadores y estudios que afirman que el juego se encuentra ya presente en los últimos meses de embarazo, dentro del vientre de sus madres. Y es que, "el juego es una de las manifestaciones más habituales en el ser humano desde su nacimiento. El niño, antes incluso de dar sus primeros pasos, tiende a buscar juegos rudimentarios que le producen una sensación de bienestar interno". ${ }^{20}$

Por otro lado, considero importante resaltar, que el juego no se trata de una metodología innovadora, sino que por el contrario es una herramienta que siempre ha estado presente en el desarrollo de la especie humana. Es más, si seguimos una teoría evolucionista darwiniana, el hombre sería la evolución de una especie animal. Pues bien, la etología, que estudia la conducta animal, confirma que el juego está presente en prácticamente todas las especies de animales ${ }^{21}$ demostrando que el juego es una conducta muy importante para su desarrollo, y que por tanto es una conducta presente para todos

\footnotetext{
${ }^{14}$ Garry Landreth, La terapia del juego (Barcelona: Obelisco, 2018).

15 Natalia Bernabeu y Andy Goldstein, Creatividad y aprendizaje. El juego como herramienta pedagógica (Madrid: Narcea, 2018).

${ }^{16}$ Carlos García, "La importancia del juego y el desarrollo de las habilidades sociales", en Raquel Monroy (coord.), Manual Práctico de Logopedia (Valencia: Psylicom, 2018).

17 Víctor Del Toro, "El juego como herramienta educativa del educador social en actividades de animación sociocultural y de ocio y tiempo libre con niños con discapacidad", Revista de Educación Social, núm. 16 (enero, 2013): 1-13.

18 Charlie Steffens y Spencer Gorin, Cómo fomentar las actitudes de convivencia a través del juego (Barcelona: CEAC, 1999).

${ }^{19}$ Raúl Bermejo, Ser maestro (Plataforma editorial, 2017).

${ }^{20}$ Gema Sáez y Antonio Monroy, "Evolución del juego a lo largo de la historia”, EFdeportes, núm. 143 (abril, 2010).

${ }^{21}$ Cley McLean, The power of play. [¿Por qué jugamos?] [Documental]. (Canadá: CBC, 2019).
} 
ellos. Si recopiláramos información de nuestros antepasados, veremos que en todas las civilizaciones antiguas estaba presente de una forma u otra el juego. Incluso si fuéramos más allá, los antropólogos confirman la presencia del juego en la prehistoria.

El juego en las escuelas debe ser un vehículo facilitador y transformador de la educación ${ }^{22}$ sobre el que se cimente la inclusión, además de ser un medio de exploración y descubrimiento para los niños y niñas. ${ }^{23}$ También es importante destacar que cualquier niño o niña disfruta jugando y evoluciona mediante el juego, ${ }^{24}$ por lo que si tiene dificultad en interaccionar en el recreo, además de mostrar conductas disruptivas o de aislamiento, se estarían perdiendo momentos de ocio y oportunidades de desarrollo para este alumnado que lo sufre. Y es que, "el juego libre resulta crucial para que una persona llegue a ser socialmente competente, maneje el estrés y desarrolle habilidades cognitivas". ${ }^{25}$ En la antigüedad se ha usado el término juego como sinónimo de diversión; pero las últimas investigaciones muestran la importancia del juego en el desarrollo de cualquier persona. Cada vez son más las metodologías educativas que incluyen el juego en los procesos de enseñanza-aprendizaje, mostrando unos resultados muy positivos. Es más, existen estudios de niños en situación de privación de juego en la infancia, en los que se observan deterioros sociales, emocionales y cognitivos derivados de la ausencia del juego en edades tempranas.

\section{Antecedentes}

Muchas veces sigue estando presente en las escuelas la duda de si el alumnado que se encuentra escolarizado en las aulas es igual o diferente entre sí; y más concretamente si todos deben o tienen que aprender de la misma manera, y por tanto utilizar una única metodología de enseñanza-aprendizaje en el aula, igual para todos y todas.

Si analizáramos detenidamente cualquier aula, en un primer golpe de vista observaríamos que físicamente ya existen grandes diferencias entre el alumnado allí presente. ${ }^{26}$ Existen rasgos físicos dispares, que hacen que podamos diferenciarnos entre

\footnotetext{
${ }^{22}$ Inma Marín, ¿Jugamos? (Barcelona: Paidós, 2018).

${ }^{23}$ André Stern, Jugar (Albuixech. España: Litera, 2017).

${ }^{24}$ García, ¿A qué jugamos?

${ }^{25}$ Melinda Wenner, "La importancia de jugar", Investigación y ciencia. Mente \& Cerebro, núm. 69 (enerofebrero 2011): 38-45.

${ }^{26}$ Carlos García-Junco, Real Oviedo Genuine. Una historia con orgullo, valor y garra (Oviedo: Gofer, 2020).
} 
nosotros y tener unas características propias como humanos que nos hacen ser diferentes. Entonces, después de este primer cribado visual en un aula, nos preguntamos: ¿Todo nuestro alumnado es igual? No, el alumnado es diferente entre sí, y es cuando surge el concepto 'diversidad'. Diversidad significa un conjunto de cosas diversas. Diverso hace referencia a que algo es diferente, que no se parece.

El problema radica en que, si en nuestras aulas tenemos alumnado diferente, con características intrínsecas y extrínsecas propias, y por tanto con potenciales y necesidades de aprendizaje diferentes, ¿cómo es posible que sigamos como docentes utilizando métodos de enseñanza-aprendizaje iguales para todos?

La escuela tiene que evolucionar a la vez que lo hace la sociedad. Estamos en una época en la que la sociedad en general avanza a un ritmo vertiginoso, y en cambio la educación, parece en ocasiones estancarse en ese proceso evolutivo. No es lógico que todo avance y los métodos de enseñanza-aprendizaje queden anclados con metodologías del pasado.

Por otro lado, y asociado a la metodología y el proceso de enseñanza-aprendizaje, no sirve de nada dotar a las aulas de los mejores aparatos tecnológicos y de las últimas tendencias innovadoras, si el profesorado no está formado, ya que no conseguirá obtener el mayor rendimiento de ellas, ni potenciar al máximo las capacidades de cada alumno, y ese tendría que ser el objetivo principal de introducir las TIC en las escuelas.

Relacionado con la diversidad, el profesorado debe estar preparado para poder detectar las dificultades que pueden aparecer entre el alumnado presente en sus clases, e intentar al menos, buscar soluciones a los problemas con el objetivo de que el alumno pueda alcanzar los objetivos académicos igualmente que sus compañeros.

Siguiendo con el término de diversidad, encontramos alumnos y alumnas con diagnósticos dispares y diversos, entre los que podemos rescatar al alumnado con TEA (Trastorno del Espectro del Autismo). Este alumnado presenta características muy diferentes y heterogéneas entre sujetos con un mismo dictamen o diagnóstico clínico, pero en general; y en el ámbito educativo pueden presentar dificultades similares en algunas áreas. Una triada que se puede estar presente en el alumnado con TEA son las dificultades en la interacción y las relaciones sociales, la comunicación y la imaginación e inflexibilidad de pensamiento. 
El alumnado con TEA presenta dificultad para comprender y respetar normas que gobiernan las relaciones con los demás y las normas sociales. Muchos alumnos y alumnas con TEA fracasan a la hora de integrarse durante los tiempos de juego o recreo con sus iguales debido a que sus métodos para relacionarse no se rigen por conductas sociales apropiadas y/o ajustadas. Sus formas de contacto social no suelen estar adaptadas, por lo que es muy común, que fracasen en las relaciones con sus compañeros, ocasionando por tanto períodos de aislamiento. Cuando son pequeños, serán los otros niños, los que no entiendan sus conductas, y esa falta de comunicación verbal y no verbal, acabará repercutiendo en un aislamiento de los niños con TEA durante las actividades de recreo o juegos compartidos durante su estancia en el colegio. A medida que avancen en edad, este aislamiento será siendo cada vez mayor, fruto del desarrollo madurativo verbal y comunicativo del resto de alumnado sin NEE, y que irá acrecentándose en el alumnado con TEA que no trabaje estas áreas.

Para buscar una posible solución a esta situación de aislamiento y exclusión social y educativa anteriormente citada en los periodos de recreos, se crea el programa Patios y Parques Dinámicos, ${ }^{27}$ que busca mediante el juego, proporcionar una herramienta inclusiva que permita que todo el alumnado disfrute de los momentos de recreo educativo. Esta herramienta y metodología se ha implementado en múltiples colegios obteniendo resultados positivos, pero en muchos de los casos sin realizar una evaluación exhaustiva, simplemente recibiendo un feedback positivo por parte del profesorado; así como generando un aumento en el interés y en la autoestima del propio alumnado. ${ }^{28}$ Siguiendo en la línea de esta propuesta inclusiva, podemos confirmar también los resultados positivos obtenidos en otros estudios que han utilizado una metodología observacional, ${ }^{29}$ destacando el aumento en interacción positiva en el juego cuando los monitores y/o profesorado se encuentra presente en el patio. En otros estudios ${ }^{30}$ se puede comprobar que la propuesta de recreos activos en

\footnotetext{
${ }^{27}$ Lagar, Patios.

28 Nuria Garrido, Recreos educativos inclusivos ;Yo también juego! (Trabajo fin de grado) (Oviedo: Universidad de Oviedo, 2020).

${ }^{29}$ Andrés García-Gómez, Miriam Ambrosio y Lidia Gil, "Intervenciones para mejorar el juego de los niños con autismo en el patio de recreo", Estudios sobre educación, núm. 38 (febrero 2020): 253-278. https://doi.org/10.15581/004.38.253-278.

30 Antonio Méndez-Giménez y Miguel Pallasá-Manteca, "Disfrute y motivación en un programa de recreos activos", Apunts. Educación Física y Deportes, núm. 134, (enero, 2018): 55-68.
} 
los centros escolares sirve como herramienta para aumentar la motivación, el disfrute y la participación del alumnado en los patios de recreo.

Además, se han desarrollado otras técnicas y herramientas digitales que se pueden utilizar de apoyo y recurso para la mejora de la interacción del alumnado con TEA en los períodos de juego; y que por tanto se pueden realizar en el recreo y ser de utilidad para mejorar la interacción e inclusión del alumnado en riesgo de exclusión en los patios escolares. Una de estas herramientas digitales es la App "TEAyudo a Jugar", ${ }^{11}$ disponible su descarga en la página web de la Fundación Orange, la cual es una herramienta tecnológica de aprendizaje, intervención e inclusión social para personas con TEA (Trastorno del Espectro del Autismo) u otras diversidades del neurodesarrollo. La app, disponible para móviles y tablets, propone momentos de juego de manera visual y permite crear situaciones de juego adaptadas al interés de cada usuario, así como una anticipación visual de las normas de los juegos que se desarrollarán en el espacio de recreo. Otra herramienta tecnológica eficaz, que en este caso puede ser de utilidad para evaluar las situaciones de exclusión social que se producen en los patios de recreo, es el programa Hoissan 1.2. ${ }^{32}$ para mejorar la recogida de datos, análisis de los mismos y poder obtener una mayor fiabilidad en estudios que utilizan una metodología observacional en contextos naturales.

\section{Propuesta}

Jugar es un acto propio de los niños y niñas. A través del juego, los niños y niñas desarrollan múltiples aspectos comunicativos y sociales. Pero en las escuelas actuales, es muy frecuente, observar alumnos/as totalmente aislados, por lo que surge la necesidad de intervenir para revertir dicha situación. Entre las necesidades de los niños y niñas con TEA se encuentran, como se ha mencionado anteriormente, las dificultades que presenta este alumnado para relacionarse y mantener habilidades sociales productivas con sus iguales. Por tanto, el alumnado con TEA, durante su jornada educativa, además de tener que trabajar con especialistas de pedagogía terapéutica y audición y lenguaje aspectos

\footnotetext{
${ }^{31}$ Lagar, Patios.

32 Antonio Hernández et al., "Hoisan 1.2: programa informático para uso en metodología observacional", Cuadernos de Psicología del Deporte, núm. 12 (junio, 2012), 55-78.
} 
curriculares debido a sus dificultades académicas, se encuentra inmerso en situaciones comunicativas nuevas e imprevistas en las que fracasa. Éstos son aspectos que se deberían de trabajar y abordar, desde los especialistas del centro educativo, y por parte de todo el profesorado que se encuentra en contacto con el alumno en cuestión.

Una de las situaciones más problemáticas que podemos encontrar para el alumnado con TEA dentro de su jornada escolar, es el recreo, la cual se produce diariamente, observándose resultados de interacción muy bajos. Para intentar mejorar esta situación se propone el proyecto ¿A qué jugamos? ${ }^{33}$ desarrollado en un centro educativo ordinario en la localidad de Oviedo. Sus dificultades en habilidades sociales y comunicativas, sumado a sus dificultades académicas, hacen que sean niños con altos porcentajes de fracaso escolar, además de sumarse otro factor de riesgo social: La exclusión social y el acoso escolar o bullying. Referente al acoso escolar o bulliyng, son niños que pueden ser maltratados al mostrarse indefensos ante situaciones violentas. Como afirma Uruñuela "la escuela es un lugar especial para el aprendizaje de la convivencia, pero no siempre tienen lugar prácticas de convivencia en positivo". ${ }^{34}$ Todos somos conscientes o hemos vivido situaciones de acoso escolar en la escuela, que en el caso de niños con TEA pueden agravar una intervención educativa, más si ésta se trata de aspectos relacionados con el juego y que necesitan del contacto con compañeros de colegio. En el alumno con TEA acosado, pueden aparecer miedos, situaciones de rechazo social, conductas de evitación, ansiedad, etc.; que dificultarían más si cabe la intervención, aumentando los tiempos de aislamiento durante los recreos de este alumnado.

El proyecto ¿A Qué Jugamos? se fundamenta en el programa de Lagar ${ }^{35}$ Patios y Parques Dinámicos, e intenta potenciar habilidades sociales adecuadas a todo el alumnado que presente conductas disruptivas en el juego con iguales. En el proyecto se fijan una serie de objetivos a conseguir:

Principal

- Desarrollar Habilidades Sociales y Comunicativas en el alumnado con Trastorno del Espectro Autista (TEA) en el entorno educativo y tiempo de recreo.

\footnotetext{
${ }^{33}$ Carlos García, ¿A qué jugamos? Inclusión del alumnado con TEA en el tiempo de recreo en centros escolares (Valencia: Psylicom, 2018).

${ }^{34}$ Uruñuela, Trabajar la convivencia, 55.

${ }^{35}$ Lagar, Patios.
} 


\section{Secundarios}

- Incluir al alumnado con TEA en todas las actividades de ocio y juego desarrolladas dentro del espacio educativo.

- Optimizar y desarrollar sus interacciones comunicativas.

- Favorecer la inclusión en las actividades grupales de aula.

- Desarrollar apoyos naturales por parte de otros niños/as.

- Sensibilizar a toda la comunidad educativa sobre las características del alumnado TEA, y la importancia de la inclusión de los alumnos con NEAE.

- Disminuir el riesgo de acoso escolar y exclusión social.

El proyecto se lleva a cabo, inicialmente, por los maestros especialistas de pedagogía terapéutica y audición y lenguaje, aunque lo ideal de este proyecto, y la propuesta futura del mismo, es la implicación por parte de todo el profesorado encargado de la vigilancia de patio.

Al estar dividido el patio, y delimitada la zona de juego por cursos, los especialistas de audición y lenguaje y pedagogía terapéutica, se reparten por cursos también, con el objetivo de registrar e intervenir con todos aquellos alumnos con TEA que se encuentran en el colegio durante el recreo de Educación Primaria. Por tanto, uno de ellos se encarga de la zona de juego de $1^{\mathrm{o}}$ y $2^{\circ}$ de Educación Primaria; y el otro de la zona de recreo de $3^{\circ}$ a $6^{\circ}$ de Educación Primaria.

El esquema de desarrollo del proyecto ¿A Qué Jugamos? es el siguiente: observación directa y registro: en esta fase, el objetivo es realizar una observación sistemática de la actividad individual de los niños con TEA durante el recreo. Esta observación es necesaria para identificar el punto de partida de cada niño, ya que es importante resaltar y recordar, que no existen niños con características y gustos para el juego iguales, por lo que la intervención y el registro se deberá realizar de forma individual.

La temporalización para este apartado se fijará dependiendo del número de sesiones dedicada a la intervención directa de cada alumno, pero serán necesarios al menos cuatro registros de cada alumno (aunque son aconsejables más) para poder comprobar las 
conductas observables en el alumno (manías, estereotipias, participación en el juego, gustos y preferencias, compañeros de juego, espacios por los que se desenvuelve, etc.).

También es recomendable que exista más de un observador, para poder generalizar el registro de las observaciones, y realizar comparaciones entre los resultados obtenidos: intervención y registro: una vez registradas las conductas y actividades del niño con TEA durante el tiempo de recreo, procederemos a analizar dichos registros, y programar actividades y juegos cuyo objetivo es el de integrar al alumno en el juego y dinámicas de patio con sus iguales.

Serán al principio situaciones de juego muy estructuradas, con apoyos visuales claros y claves de trabajo concisas, tanto para el alumno en cuestión, como para el resto de los alumnos y compañeros, y también para el profesorado encargado de llevar a cabo las actividades y/o juegos.

La rutina de juego consiste en que "al principio el maestro/a deberá mediar y dirigir los juegos a realizar. Llegará un momento, en el que la iniciativa la tomen los propios alumnos/as y sean éstos, los que dirijan los juegos". 36

El tipo de investigación seleccionado para evaluar el proyecto ¿A Qué Jugamos?, y, por tanto, analizar la inclusión de un caso único de niño con TEA en el recreo en un centro educativo ordinario, es la investigación experimental de caso único, utilizando una metodología de carácter observacional, que puede proporcionar soluciones metodológicas fiables para la obtención de datos en ausencia de herramientas de evaluación estandarizadas. ${ }^{37}$

El diseño de caso único constituye, en la actualidad, una herramienta de trabajo con gran aceptación dentro del ámbito de las ciencias sociales y de la salud. ${ }^{38}$ También otros autores $^{39}$ afirman que la metodología observacional posibilita el análisis del conjunto de interacciones y conductas del alumno, tanto en el aula como en cualquier contexto de

\footnotetext{
${ }^{36}$ Francisco Luis Aranda et al., Manual práctico para alumnado con TEA. Rutinas y Talleres (Valencia: Psylicom, 2013).

37 Mariona Portell et al., "Guidelines for Reporting Evaluations Based on Observational Methodology", Psicothema, núm. 27 (abril, 2015): 283-289.

${ }^{38}$ Roser Bono y Jaume Arnau, Diseños de caso único en ciencias sociales y de la salud (Madrid: Síntesis, 2014).

${ }^{39}$ Ignacio Pedrosa, África Borges, Natalia Herranz, Mayurena Lorenzo y Eduardo García-Cueto, "Desarrollo del Protocolo de Observación de interacción en el Aula: aplicación en un programa de niños con altas capacidades", Revista de Educación, Extraordinario (marzo, 2013): 293-312.
} 
enseñanza no formal; por tanto es una metodología muy válida para este tipo de estudio, ya que observaremos las conductas de un alumno con TEA en un contexto de enseñanza no formal, como es el patio de recreo del colegio.

Además, se busca un sistema observacional que sea idéntico y que sirva para registrar las fases de observación y tratamiento, para posteriormente, comparar ambos períodos, con el fin de visualizar los resultados y poder identificar posibles avances o retrocesos y realizar unas conclusiones sobre la interacción del niño con TEA objeto de estudio. El estudio se realiza mediante el sistema de medidas repetidas realizadas por diferentes observadores. Lo que se registra, son los tiempos de las diferentes conductas, tanto de interacción, como de aislamiento, observadas en el niño con TEA y cuantificados en minutos que se distribuyen en pequeños grupos de 5 minutos. La idea futura es idear un sistema informático más exhaustivo que permita evaluar y registrar minutos y segundos con una mayor exactitud de las conductas observadas y realizadas por el alumnado con TEA durante los recreos.

La secuenciación de fases o períodos viene determinada por la introducción o retirada de un tratamiento. En el caso del proyecto ¿A Qué Jugamos?, la primera fase, o línea base, es la observación y registro de las conductas del alumno con TEA en el recreo sin la introducción del tratamiento, que, en este caso, es la puesta en marcha del proyecto, facilitando la interacción en el juego con otros niños mediante juegos propuestos. La siguiente fase, es la del tratamiento y registro, (denominada fase de tratamiento o experimental) de las conductas del alumno una vez introducidas las modificaciones, las cuales son, la facilitación de juegos con iguales. Para el presente estudio se han fijado 10 sesiones para cada fase en un modelo $\mathrm{ABAB}$ por considerar que quizás es el que más se puede ajustar a la realidad educativa que vivencia el alumnado con TEA en los centros educativos y que puede dotar a los resultados obtenidos de una mayor fiabilidad.

Para la elaboración y definición de categorías del registro observacional (Anexo 1), se ha tomado como referencia el trabajo de autores previos ${ }^{40}$ adaptando y ajustando las conductas al alumnado con TEA:

${ }^{40}$ Ibid., 301. 
Categoría 1: Interacción: recoge aquellas conductas y/o comportamientos del alumno en relación con la interacción con otros niños o niñas.

- Interacción positiva: incluye todos aquellos comportamientos que sirven para relacionarse de manera adecuada con los compañeros o con el profesor por iniciativa propia o como respuesta a una interacción de los otros.

- Interacción negativa: recoge los comportamientos incorrectos del alumno hacia sus iguales.

Categoría 2: Aislamiento: recoge aquellas conductas y/o comportamientos del alumno con relación al aislamiento.

- Aislamiento neutro: el alumno se aísla del contacto con sus iguales sin producir conductas disruptivas o inadaptadas.

- Aislamiento negativo: el alumno se aísla del contacto con sus iguales produciendo conductas disruptivas o inadaptadas.

Categoría 3: Instrumental: recoge los comportamientos y conductas del alumno que no se corresponden con ninguna de las categorías anteriores.

En este estudio para el registro se podría decir que se optó por una modalidad mixta en cuanto a la participación de los observadores en la recogida de datos. Por una parte, en la primera fase (A) o línea base, se llevó a cabo una observación externa o no participante, en la que los observadores se mantenían pasivos, como si fueran espectadores, de la situación que ocurría en el recreo. Su labor durante esta fase únicamente consistía en registrar las conductas observadas durante el recreo del alumno con TEA. En cambio, en la segunda fase (B) o fase de tratamiento, los observadores adoptan una posición participante, ya que son ellos los encargados además de recoger los datos de las conductas observadas, de proponer los juegos y vigilar las zonas de juego acotadas en el patio durante los recreos.

\section{Resultados}

Además de los registros obtenidos por los diferentes observadores se ha optado por calcular los índices del tamaño del efecto del estudio con el método PND (Percentage of Non-Overlapping Data). Para el análisis de los resultados obtenidos se generan además una serie de gráficos 
divididos por categorías que permiten una mejor interpretación visual del proyecto. Los análisis completos se pueden encontrar en la publicación que se especifica a pie de página ${ }^{41}$ ya que a continuación simplemente se muestran aquellos que se han considerado:

Interacción: se puede observar como la mejora en la interacción es cuantificable en aquellas fases en las que el proyecto se realiza y está presente en los recreos.
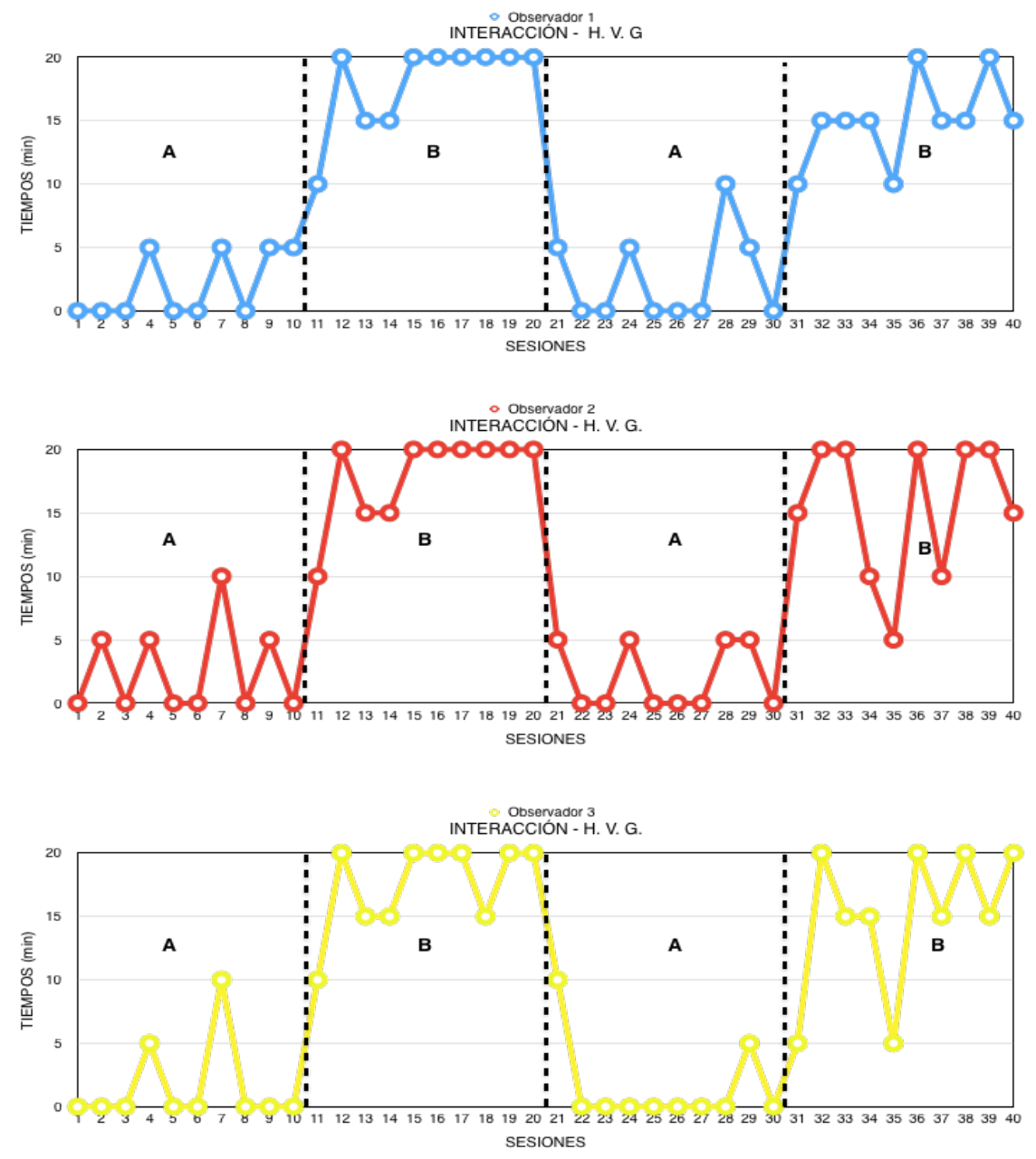

\begin{tabular}{|c|c|l|}
\hline \multicolumn{3}{|c|}{ PND - Interacción } \\
\hline Observador 1 & $17 / 20=85 \%$ & Bastante efectivo \\
\hline Observador 2 & $16 / 20=80 \%$ & Bastante efectivo \\
\hline Observador 3 & $17 / 20=85 \%$ & Bastante efectivo \\
\hline TOTAL & $50 / 60=83 \%$ & Bastante efectivo \\
\hline
\end{tabular}

${ }^{41}$ García, ¿A qué jugamos? 
Interacción con alumnado de su grupo clase: se observa en los gráficos una mejoría considerable en comparación a la línea base. Además, los resultados del cálculo del PND reconocen la efectividad de la introducción del programa.
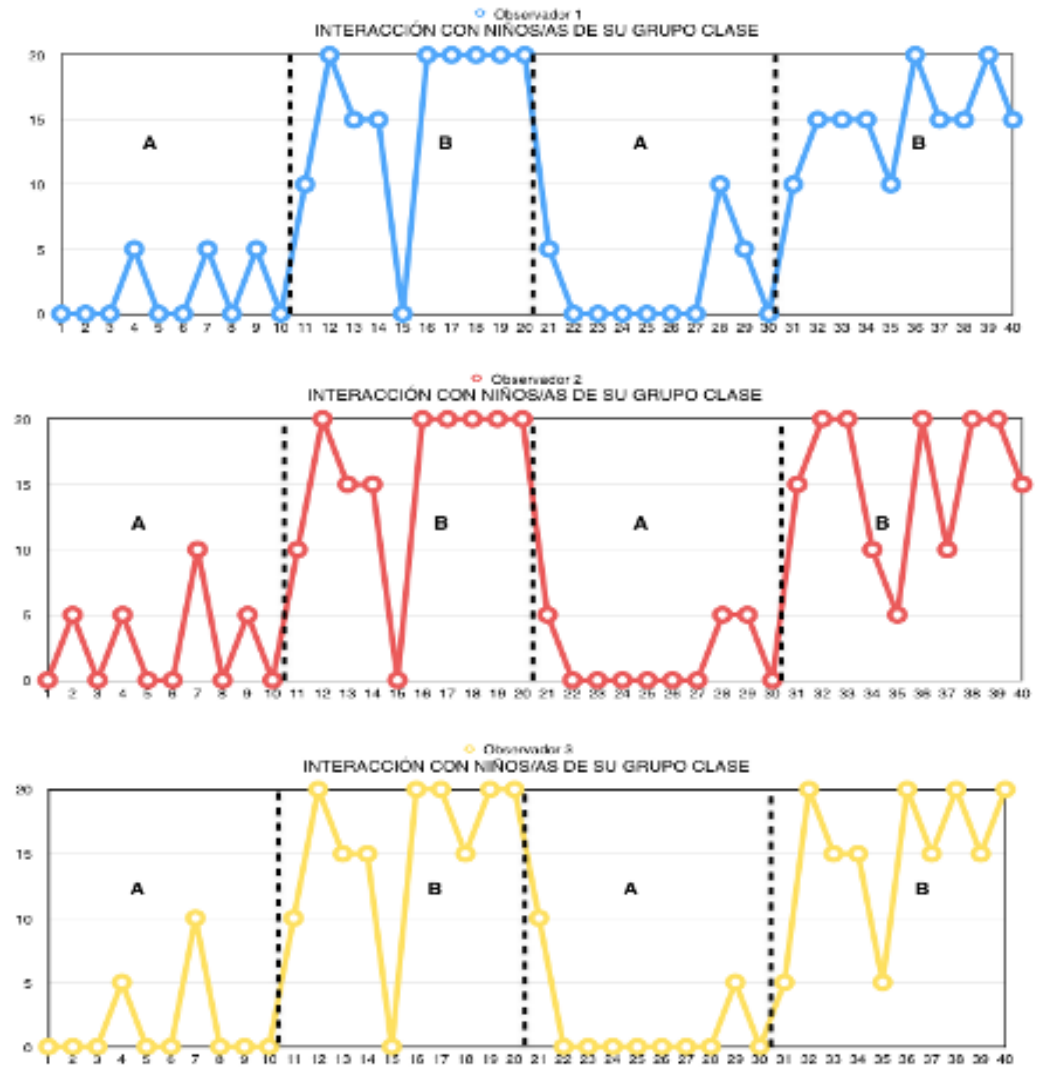

\begin{tabular}{|c|c|c|}
\hline \multicolumn{3}{|c|}{ PND - Interacción con niños/as grupo clase } \\
\hline Observador 1 & $16 / 20=80 \%$ & Bastante efectivo \\
\hline Observador 2 & $15 / 20=75 \%$ & Bastante efectivo \\
\hline Observador 3 & $16 / 20=80 \%$ & Bastante efectivo \\
\hline TOTAL & $47 / 60=78 \%$ & Bastante efectivo \\
\hline
\end{tabular}

Interacción con alumnado de otras aulas: se observa en los gráficos una mejoría considerable en comparación a la línea base. Aunque como vemos en la tabla de debajo, los resultados del PND, no son tan óptimos como los recogidos con los niños de su grupo clase. Se observa un aumento en los tiempos de interacción con niños de otras aulas, aunque también en este aspecto, podemos destacar que dicha interacción no se mantiene durante toda la intervención, pero sí se dan días de intervención en los que la interacción aumenta con niños de otras aulas (que para él en la mayoría de los casos son desconocidos). 

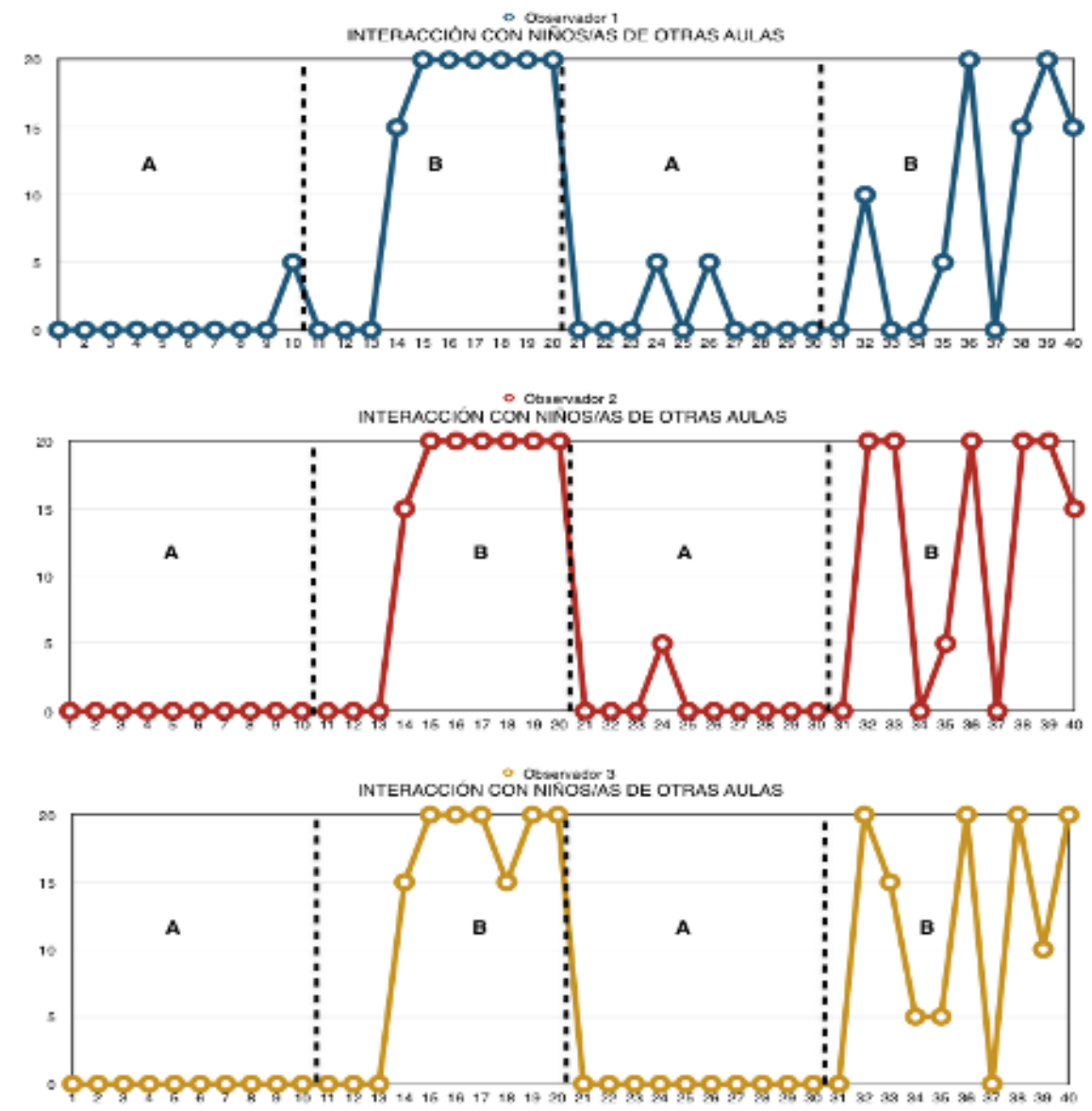

\begin{tabular}{|c|c|c|}
\hline \multicolumn{3}{|c|}{ PND - Interacción con niños/as otras aulas } \\
\hline Observador 1 & $12 / 20=60 \%$ & Efectividad cuestionable \\
\hline Observador 2 & $13 / 20=65 \%$ & Efectividad cuestionable \\
\hline Observador 3 & $16 / 20=80 \%$ & Bastante efectivo \\
\hline TOTAL & $41 / 60=68 \%$ & Efectividad cuestionable \\
\hline
\end{tabular}

Manías: uno de los aspectos que más preocupaban en cuanto a las conductas del alumno objeto de estudio, era la presencia de manías durante el recreo; ya que este alumno no se relacionaba con otros niños/as y además presentaba manías como perseguir palomas o abrir y cerrar puertas. Una vez finalizada la investigación podemos confirmar que este aspecto ha sido mejorado con la puesta en marcha del proyecto ¿A Qué Jugamos?, siendo por tanto esta intervención bastante efectiva en esta conducta. 

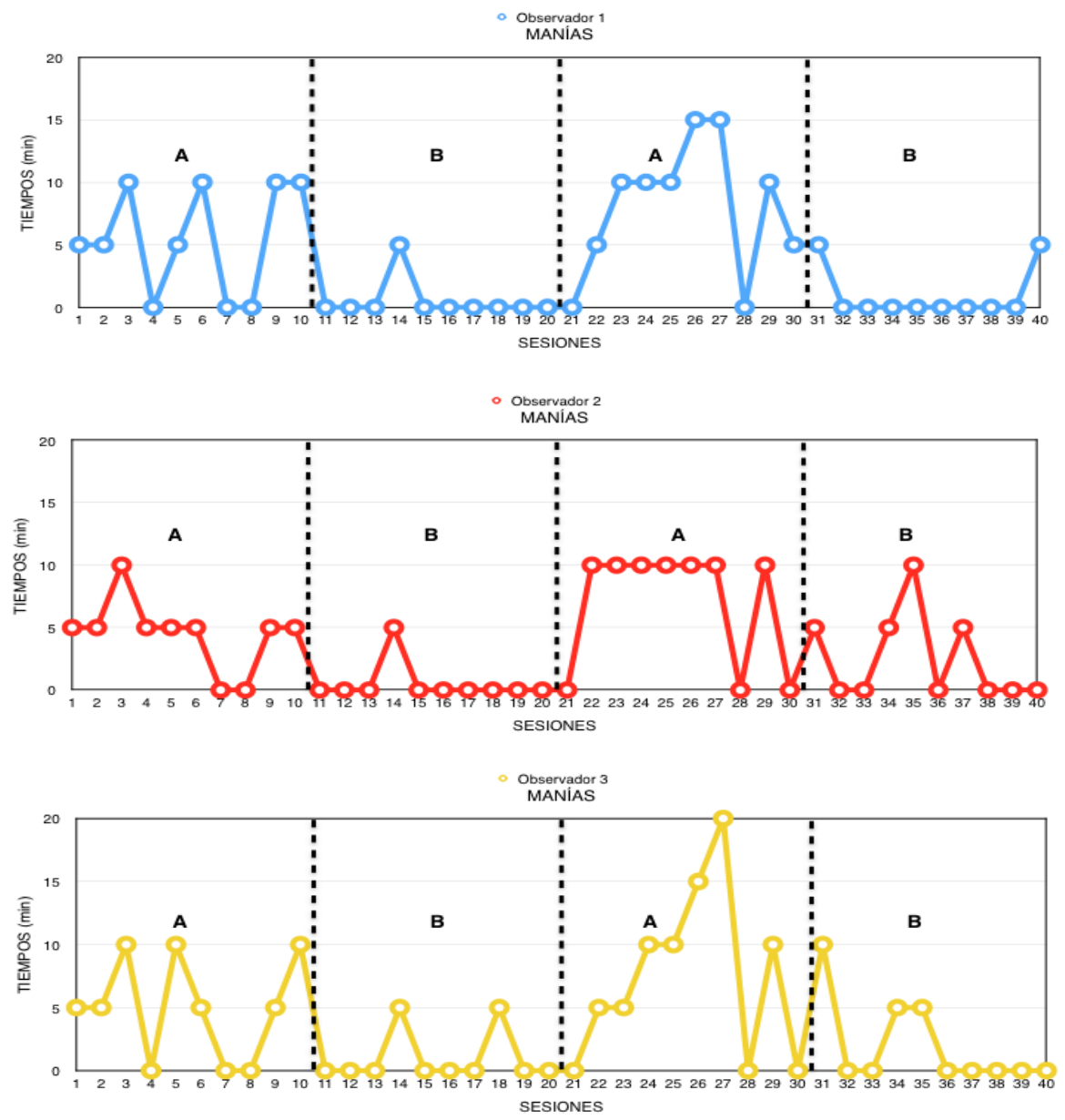

\begin{tabular}{|c|c|l|}
\hline \multicolumn{3}{|c|}{ PND - Manías } \\
\hline Observador 1 & $17 / 20=85 \%$ & Bastante efectivo \\
\hline Observador 2 & $15 / 20=75 \%$ & Bastante efectivo \\
\hline Observador 3 & $15 / 20=75 \%$ & Bastante efectivo \\
\hline TOTAL & $47 / 60=78 \%$ & Bastante efectivo \\
\hline
\end{tabular}

\section{Conclusiones}

Con el presente estudio, podemos dar validación al proyecto ¿A Qué Jugamos?, ya que mediante un análisis $\mathrm{N}=1$, se demuestra que los tiempos de interacción del alumnado con TEA aumentan en las fases de intervención respecto a los resultados obtenidos en la línea base. También confirmar la importancia de la intervención en los tiempos de recreo de los centros educativos, con el objetivo de conseguir una inclusión real y más efectiva de todo el alumnado.

Bien es cierto que no se pueden generalizar los resultados con este único estudio, ya 
que al tratarse de un único alumno con TEA con el que se ha realizado el estudio, no podemos generalizar al resto de alumnado con TEA escolarizado en otros centros educativos; pero sí podemos concluir desde nuestra experiencia, que la introducción del proyecto ha provocado una disminución de conflictos en el tiempo de recreo entre el alumnado en general, provocando por tanto, beneficios en toda la comunidad educativa.

A su vez podemos confirmar los beneficios recogidos en el trabajo Patios y Parques Dinámicos ${ }^{42}$, en el que expone que "la finalidad última que buscamos con nuestra actuación es conseguir que el niño o la niña disfrute de ese tiempo de ocio, al igual que el resto de los compañeros y compañeras". Por lo que, una vez realizado el estudio, podemos reafirmar la teoría de Gey Lagar, ya que nuestro alumno objeto de estudio, además de observarse en él un aumento de los tiempos de juego, y por tanto de interacción positiva, se ha observado de manera subjetiva por los observadores y resto de profesorado, cómo el alumno disfrutaba de la participación con sus iguales en los diferentes juegos propuestos durante los recreos.

En los ambientes educativos se suelen utilizar estudios que analizan una determinada situación de un alumno en concreto o un grupo de alumnado, pero no siempre se realizan registros sistemáticos de las actividades llevadas a cabo en contextos escolares y que pueden aportar valiosa información., por lo que como conclusión general, además de apostar firmemente por la inclusión de programas educativos en los patios escolares, expongo la necesidad de registrar y evaluar de manera sistemática y observacional las conductas que el alumnado en riesgo de exclusión social realiza durante su escolarización, con el objetivo de poder evaluar y programas acciones ajustadas a sus necesidades personales y académicas.

${ }^{42}$ Lagar, Patios. 


\section{Bibliografía}

Alcedo, María Ángeles, Laura Elisabet Gómez, Antonio León Aguado, Benito Arias y Rosalía González. "Eficacia del contacto e información como técnicas de cambio de actitudes hacia personas con discapacidad en niños de Educación Primaria". Universitas Psychologica, núm. 12 (agosto, 2012): 493-504. https://doi.org/10.11144/Javeriana.UPSY12-2.ecit.

Amor, Antonio, Miguel Ángel Verdugo y María Fernández. “Gathering Evidences in all System-levels for the Applied Impact of Disability Studies on People with Disabilities Lives”. En Joanna Glodkowska, Justyna Maria Gasik y Marta Pagowska (coords.), Studies on Disability. Varsovia: Wydawnictwo Akademii Pedagogiki Specjalnej, 2018.

Aranda, Francisco Luis, Elena López, María del Carmen Marcos, Marina Morales, María del Pilar Moreno, Eva María Orellana y Francisco Javier Torres. Manual práctico para alumnado con TEA. Rutinas y Talleres. Valencia: Psylicom, 2013.

Artavia, Jenny María. "Manifestaciones ocultas de violencia, durante el desarrollo del recreo escolar" Revista Electrónica Actualidades Investigativas en Educación, núm. 13 (enero, 2013): 1-21.

Bermejo, Raul. Ser maestro. Plataforma editorial, 2017.

Bernabeu, Natalia, y Andy Goldstein. Creatividad y aprendizaje. El juego como herramienta pedagógica. Madrid: Narcea, 2018.

Bona, Cesar. Las escuelas que cambian el mundo. Barcelona: Plaza \& Janés, 2016.

Bono, Roser, y Jaume Arnau. Diseños de caso único en ciencias sociales y de la salud. Madrid: Síntesis, 2014.

Calderón, Ignacio, y Paula Verde. Reconocer la diversidad. Barcelona: Octaedro, 2018.

Del Toro, Víctor. "El juego como herramienta educativa del educador social en actividades de animación sociocultural y de ocio y tiempo libre con niños con discapacidad". Revista de Educación Social, núm. 16 (enero 2013): 1-13.

Echeita, Gerardo, y Ignacio Calderón-Almendros. “Obstáculos a la inclusión: cuestionando concepciones y prácticas de evaluación psicopedagógica". Ámbitos de Psicopedagogía y Orientación, núm. 41 (noviembre, 2014): 1-11.

Echeita, Gerardo, y Cecilia Simón. "Comprender la educación inclusiva para intentar 
llevarla a la práctica". En Henar Rodríguez y Luis Torrego (coordd.), Educación inclusiva, equidad y derecho a la diferencia. Madrid: Wolters Kluwer, 2013.

España. Ley Orgánica 8/2013, de 9 de diciembre, para la mejora de la calidad educativa. Boletín Oficial del Estado, 295, de 10 de diciembre de 2013, 97858-97921. http://www.boe.es/boe/dias/2013/12/10/pdfs/BOE-A-2013-12886.pdf.

García, Carlos. ¿A qué jugamos? Inclusión del alumnado con TEA en el tiempo de recreo en centros escolares. Valencia: Psylicom, 2018.

García, Carlos. "La importancia del juego y el desarrollo de las habilidades sociales". En Raquel Monroy (coord.), Manual Práctico de Logopedia. Valencia: Psylicom, 2018.

García-Junco, Carlos. Real Oviedo Genuine. Una historia con orgullo, valor y garra. Oviedo: Gofer, 2020.

García-Gómez, Andrés, Miriam Ambrosio y Lidia Gil. "Intervenciones para mejorar el juego de los niños con autismo en el patio de recreo". Estudios sobre Educación, núm. 38 (febrero, 2020): 253-278. https://doi.org/10.15581/004.38.253-278.

Garrido, Nuria. Recreos educativos inclusivos ;Yo también juego! (Trabajo fin de grado). Oviedo, 2020.

González-Gil, Francisco, Elena Martín-Pastor, Raquel Poy y Cristina Jenaro. "Percepciones del profesorado sobre la inclusión: estudio preliminar”. Revista Electrónica de Formación del Profesorado, núm. 19 (abril, 2016): 11-24, https://doi.org/10.6018/reifop.19.3.219321.

Gras, Pablo, y Jorge Paredes. “El recreo ¿Sólo para jugar?”. Revista Digital de Educación Física, núm. 36 (octubre, 2015): 18-27.

Hernández, Antonio, José Antonio López, Julen Castellano, Verónica Morales y José Luis Pastrana. "Hoisan 1.2: programa informático para uso en metodología observacional”. Cuadernos de Psicología del Deporte, núm. 12 (junio, 2012), 5578.

Lagar, Gey. Patios y parques dinámicos. Oviedo: Trabe, 2015.

Landreth, Garry. La terapia del juego. Barcelona: Obelisco, 2018.

Marín, Inma. ¿Jugamos? Barcelona: Paidós, 2018.

McLean, Cley. The Power of Play. [¿Por qué jugamos?] [Documental]. Canadá: CBC, 2019. 
Méndez-Giménez, Antonio, y Miguel Pallasá-Manteca. "Disfrute y motivación en un programa de recreos activos". Apunts. Educación Física y Deportes, núm. 134 (enero, 2018): 55-68.

Nacimiento, Lydia, y Joaquín Antonio Mora. Estrategias de afrontamiento ante bullying y cyberbullying: condicionantes y consecuencias. Sevilla: Universidad de Sevilla, 2018.

Pedrosa, Ignacio, África Borges, Natalia Herranz, Maryurena Lorenzo y Eduardo GarcíaCueto. "Desarrollo del Protocolo de Observación de interacción en el Aula: aplicación en un programa de niños con altas capacidades". Revista de Educación, Extraordinario (marzo, 2013): 293-312.

Portell, Mariona, María Teresa Anguera, Salvador Chacón-Moscoso y Susana SanduveteChaves. "Guidelines for Reporting Evaluations Based on Observational Methodology". Psicothema, núm. 27 (abril, 2015): 283-289.

Sáez, Gema, y Antonio Monroy. "Evolución del juego a lo largo de la historia". EFdeportes, núm. 143 (abril, 2010).

Steffens, Charlie, y Spencer Gorin. Cómo fomentar las actitudes de convivencia a través del juego. Barcelona: CEAC, 1999.

Stern, André. Jugar. Albuixech. España: Litera, 2017.

Torres, Belkys Edith. Sistema CEDA: Evaluación alternativa para una enseñanza inclusiva (tesis doctoral). Oviedo: Universidad de Oviedo, 2017.

Uruñuela, Pedro María. Trabajar la convivencia en los centros educativos. Madrid: Narcea, 2016.

Wenner, Melinda. "La importancia de jugar". Investigación y ciencia. Mente \& Cerebro, núm. 69 (enero-febrero, 2011): 38-45. 


\section{Anexo 1}

DESCRIPCIÓN DE LAS CATEGORÍAS DEL ANÁLISIS OBSERVACIONAL

\begin{tabular}{|c|c|c|c|}
\hline CATEGORÍAS & \multicolumn{2}{|c|}{ CÓDIGOS } & DESCRIPCIÓN \\
\hline \multirow{6}{*}{ 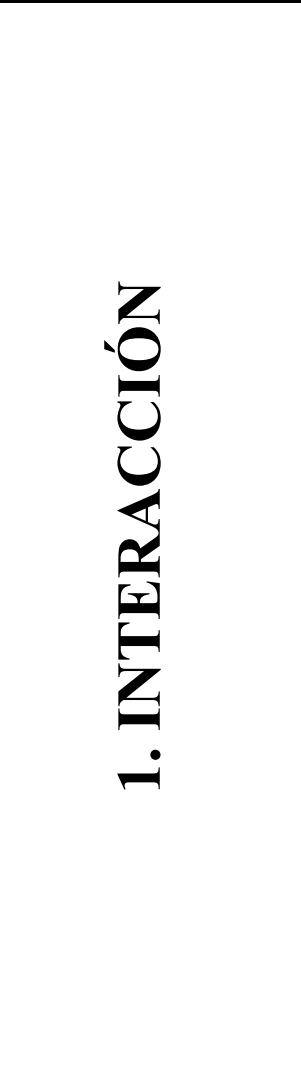 } & \multirow{4}{*}{$\begin{array}{l}1.1 . \\
\text { INTERACCIÓN } \\
\text { POSITIVA }\end{array}$} & $\begin{array}{l}\text { 1.1.1. Habla con su } \\
\text { grupo clase }\end{array}$ & $\begin{array}{l}\text { El alumno inicia o mantiene una } \\
\text { conversación con un compañero/a de } \\
\text { su grupo clase. }\end{array}$ \\
\hline & & $\begin{array}{c}\text { 1.1.2. Habla con } \\
\text { otras aulas }\end{array}$ & $\begin{array}{l}\text { El alumno inicia o mantiene una } \\
\text { conversación con un niño o niña de } \\
\text { otra aula. }\end{array}$ \\
\hline & & $\begin{array}{l}\text { 1.1.3. Juega con su } \\
\text { grupo clase }\end{array}$ & $\begin{array}{l}\text { El alumno participa en juegos } \\
\text { compartidos con un compañero/a de su } \\
\text { grupo clase. }\end{array}$ \\
\hline & & $\begin{array}{c}\text { 1.1.4. Juega con } \\
\text { otras aulas }\end{array}$ & $\begin{array}{l}\text { El alumno participa en juegos } \\
\text { compartidos con un niño o niña de otra } \\
\text { aula. }\end{array}$ \\
\hline & \multirow{2}{*}{$\begin{array}{c}1.2 . \\
\text { INTERACCIÓN } \\
\text { NEGATIVA }\end{array}$} & $\begin{array}{l}\text { 1.2.1. Agresión con } \\
\text { su grupo clase }\end{array}$ & $\begin{array}{l}\text { El alumno agrede, física o } \\
\text { verbalmente, a uno o varios de sus } \\
\text { compañeros/as de su grupo clase. }\end{array}$ \\
\hline & & $\begin{array}{c}\text { 1.2.2. Agresión con } \\
\text { otras aulas }\end{array}$ & $\begin{array}{l}\text { El alumno agrede, física o } \\
\text { verbalmente, a uno o varios niños o } \\
\text { niñas de otra aula. }\end{array}$ \\
\hline \multirow{5}{*}{$Z_{E}$} & \multirow{3}{*}{$\begin{array}{c}2.1 . \\
\text { AISLAMIENTO } \\
\text { NEUTRO }\end{array}$} & 2.1.1. Profesorado & $\begin{array}{l}\text { El alumno se junta al grupo de } \\
\text { profesores encargados de la vigilancia } \\
\text { de patio. }\end{array}$ \\
\hline & & 2.1.2. Camina & $\begin{array}{l}\text { El alumno camina y/o deambula por el } \\
\text { patio sin establecer contacto físico o } \\
\text { verbal con iguales. }\end{array}$ \\
\hline & & 2.1.3. Banco & $\begin{array}{l}\text { El alumno se sienta en uno de los } \\
\text { bancos del patio de recreo sin } \\
\text { establecer contacto físico y/o verbal } \\
\text { con iguales }\end{array}$ \\
\hline & \multirow{2}{*}{$\begin{array}{c}2.2 . \\
\text { AISLAMIENTO } \\
\text { NEGATIVO }\end{array}$} & 2.2.1 Manías & $\begin{array}{l}\text { El alumno presenta manías (abre y } \\
\text { cierra puertas, persigue palomas, sigue } \\
\text { las líneas del patio, etc.) }\end{array}$ \\
\hline & & & El alumno se encierra en el baño y se \\
\hline
\end{tabular}




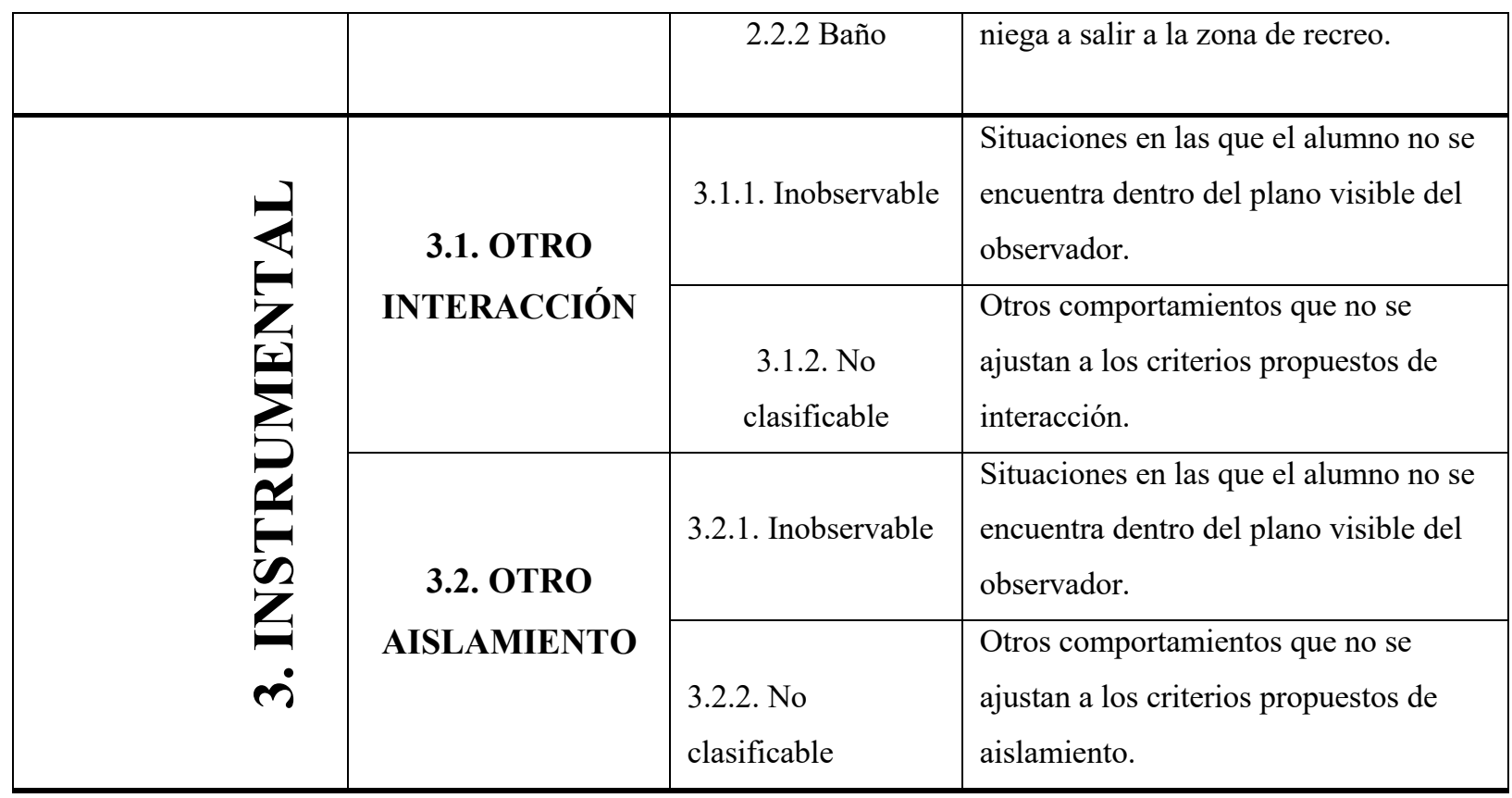

